\title{
BMJ Open Delineating the trajectories of social and occupational functioning of young people attending early intervention mental health services in Australia: a longitudinal study
}

\author{
Frank lorfino, ${ }^{1}$ Daniel F Hermens, ${ }^{1,2}$ Shane, PM Cross, ${ }^{1}$ Natalia Zmicerevska, ${ }^{1}$ \\ Alissa Nichles, ${ }^{1}$ Caro-Anne Badcock, ${ }^{3}$ Josine Groot, ${ }^{1}$ Elizabeth M Scott, ${ }^{1}$ \\ Ian B Hickie ${ }^{1}$
}

To cite: lorfino F, Hermens DF, Cross S,PM, et al. Delineating the trajectories of social and occupational functioning of young people attending early intervention mental health services in Australia: a longitudinal study. BMJ Open 2018;8:e020678. doi:10.1136/ bmjopen-2017-020678

- Prepublication history and additional material for this paper are available online. To view these files, please visit the journal online (http://dx.doi. org/10.1136/bmjopen-2017020678).

Received 16 November 2017 Revised 6 February 2018 Accepted 16 February 2018

Check for updates

${ }^{1}$ Youth Mental Health Team, Brain and Mind Centre, The University of Sydney, Sydney, New South Wales, Australia

${ }^{2}$ Mind and Neuroscience Thompson Institute, University of the Sunshine Coast, Sunshine Coast, QLD, Australia

${ }^{3}$ Statistical Consulting, The University of Sydney, Sydney, New South Wales, Australia

Correspondence to Mr Frank lorfino;

frank.iorfino@sydney.edu.au

\section{ABSTRACT}

Objectives Mental disorders typically emerge during adolescence and young adulthood and put young people at risk for prolonged socioeconomic difficulties. This study describes the longitudinal course of social and occupational functioning of young people attending primary care-based, early intervention services. Design A longitudinal study of young people receiving mental healthcare.

Setting Data were collected between January 2005 and August 2017 from a designated primary care-based mental health service.

Participants 554 young people (54\% women) aged 12-32 years.

Measures A systematic medical file audit collected clinical and functional information at predetermined time intervals (ie, 3 months to $5+$ years) using a clinical pro forma. Group-based trajectory modelling (GBTM) was used to identify distinct trajectories of social and occupational functioning over time (median number of observations per person=4; median follow-up time=23 months).

Results Between first clinical contact and time last seen, $15 \%$ of young people had reliably deteriorated, $23 \%$ improved and $62 \%$ did not demonstrate substantive change in function. Of the whole cohort, $69 \%$ had functional scores less than 70 at time last seen, indicative of ongoing and substantive impairment. GBTM identified six distinct functional trajectories whereby over $60 \%$ had moderate-to-serious functional impairment at entry and remained chronically impaired over time; $7 \%$ entered with serious impairment and deteriorated further; a quarter were mildly impaired at entry and functionally recovered and only a small minority (4\%) presented with serious impairments and functionally improved over time. Not being in education, employment or training, previous hospitalisation and a younger age at baseline emerged as significant predictors of these functional trajectories. Conclusion Young people with emerging mental disorders have significant functional impairment at presentation for care, and for the majority, it persists over the course of clinical care. In addition to providing clinical care earlier in the course of illness, these data suggest that more

\section{Strengths and limitations of this study}

- This study used a rich dataset of 554 participants with between two and nine observations per person (median=4; approximately 2200 data points) up to 5 years after initial presentation and applied a novel group-based trajectory modelling procedure to characterise the pattern of change in functional impairment over time.

- This study is one of the first to report on the long-term functional outcomes for young people attending primary care-based, early intervention mental health services. Its naturalistic design provides valuable insight into the extent of functional impairment over the course of these common mental disorders and raises specific questions about how to improve health service and individual intervention strategies to monitor, target and improve these outcomes.

- Since this was a naturalistic cohort study, there may be some factors that account for the trajectories or differences in functional outcome that were not collected in this study, such as socioeconomic status, the type and intensity of interventions an individual received. Since these factors were not uniformly collected, it is difficult to make specific conclusions about the effect of specific intervention or service models on these trajectories or outcomes.

- Since this study focuses on individuals who were continually engaged in clinical care and represents $18 \%$ of the total research register, it is unclear how representative this sample is of the whole population presenting to these services. Similarly, there is a lack of information about the differences between those who continually engage in care versus those who may have disengaged.

sophisticated and more intensive individual-level and organisational strategies may be required to achieve significant and sustained functional improvements.

\section{INTRODUCTION}

Mental disorders consistently rank among the leading causes of death and disability worldwide. $^{1-3}$ These disorders typically emerge during adolescence and young adulthood and put these young people at 
risk for prolonged socioeconomic difficulties over their lifetime, even when their mental ill health subsides or is subthreshold. ${ }^{4-7}$ There are major direct healthcare costs attributed to diagnosis and treatment; however, it is their indirect costs linked to income loss through mortality, disability and regular absences from education or work that impact future income potential and have substantial global economic consequences. ${ }^{89}$ The significant overlap between these disorders, economic inactivity and functional impairment reiterates the need to recognise and address the common health and economic vulnerabilities of these young people. ${ }^{10}$

The long-term outcomes for most major mental disorders often include high rates of recurrence, and slow or incomplete functional recovery, even among those who may have symptomatically remitted. ${ }^{11-14}$ Long-term follow-up studies among older adults indicate that functional impairment often persists with most people experiencing some degree of disability during the majority of the long-term follow-up period, ${ }^{15}$ while it is common for those within a primary care setting to spend up to one-third of the long-term follow-up period off work. ${ }^{16}$ These patterns are also evident among young people, since most medical and psychological treatments developed to address depression do not consistently improve functioning in these populations. ${ }^{17-19}$ Of the few studies that report long-term functional outcomes for young people, most adolescents treated for depression experienced positive functional outcomes up to 3 years later; however, persistent functional impairment was common for those with comorbidity and recurrence of depression. ${ }^{20}$ Similarly, young people with psychosis tend to experience significant social disability that persists over time and may be indicative of the difficulty of achieving functional recovery in these groups. ${ }^{21}$ For many of these severe mental disorders, the onset of functional deterioration tends to occur prior to the onset of illness and suggests that there is the capacity to address these problems early. 223

Early intervention services and models of care have been designed to respond to the early phases of these disorders, their associated comorbidities and impairment, to prevent or delay the progression of illness and reduce the burden for those at risk. ${ }^{24-26}$ Although many young people present with subthreshold syndromes, they frequently report significant functional impairment (ie, reduced functioning in social, occupational or other areas of daily life) and a high rate of disengagement from education, employment or training (not in education, employment or training (NEET) ). ${ }^{24}{ }^{27-29}$ Over time, functional impairment tends to be associated with symptom remission; however, the overall level of impairment and rate of disengagement remains high compared with the community. ${ }^{30-32}$ This is particularly the case for those with more severe presentations who, despite receiving more intensive initial interventions, are unlikely to functionally recover in relatively short-term care environments. ${ }^{33}$ While the first 12 months of care are characterised by significant changes in functional impairment, ${ }^{34}$ the longterm patterns of functional impairment among young people engaged in primary mental healthcare remains largely unknown.

Understanding the changes in social and occupational functioning over time in real-world clinical cohorts is crucial for guiding the development of mental health service provisions that meet the individual needs of young people with emerging mental disorders. This study examines the longitudinal course of social and occupational functioning for a cohort of young people after their initial presentation to a primary mental healthcare service. We report on the overall rate of change in social and occupational functioning, and aim to determine whether there are distinct long-term trajectories (via modelling) of functioning over the course of care.

\section{METHODS}

\section{Participants}

Study participants were drawn from a larger longitudinal cohort of young people $(n=3087 ; 59 \%$ female, mean age $=18.52 \pm 3.8$ ) presenting to the Brain and Mind Centre's youth mental health clinics in the Sydney suburbs of Camperdown and Campbelltown. These clinics consist of an integrated mix of primary-level services branded as headspace as $^{35}$ well as more specialised services including psychiatric services. These clinics primarily attract young people with a range of mental health problems, including those with subthreshold and full-threshold mental disorders, who may have been self-referred, referred via a family member or friend or else via the community including external general practitioner, schools or university. ${ }^{29}$ The young people in this study were recruited to a research register for mood, psychotic, developmental and other mental disorders between January 2005 and August 2017. All young people received clinician-based case management and relevant psychological, social and/or medical interventions over the duration of their time in care, which may also include referral to/from higher tier mental health services or hospitalisation for those whose needs exceed the capacity of the primary care services. Individuals were included in the present study if they met the following inclusion criteria: (1) between 12 and 32 years of age at the time of initial assessment; (2) were seen by a clinician on at least two separate occasions. Exclusion criteria for all potential participants were: medical instability or lack of capacity to give informed consent (as determined by a psychiatrist), history of neurological disease (eg, tumour, head trauma, epilepsy), medical illness known to impact cognitive and brain function (eg, cancer, electroconvulsive therapy in last 3 months) and/ or clinically evident intellectual disability and/or insufficient English to participate in the research protocol.

\section{Data collection process}

Trained research psychologists and medical officers conducted a medical file audit to collect demographic, 


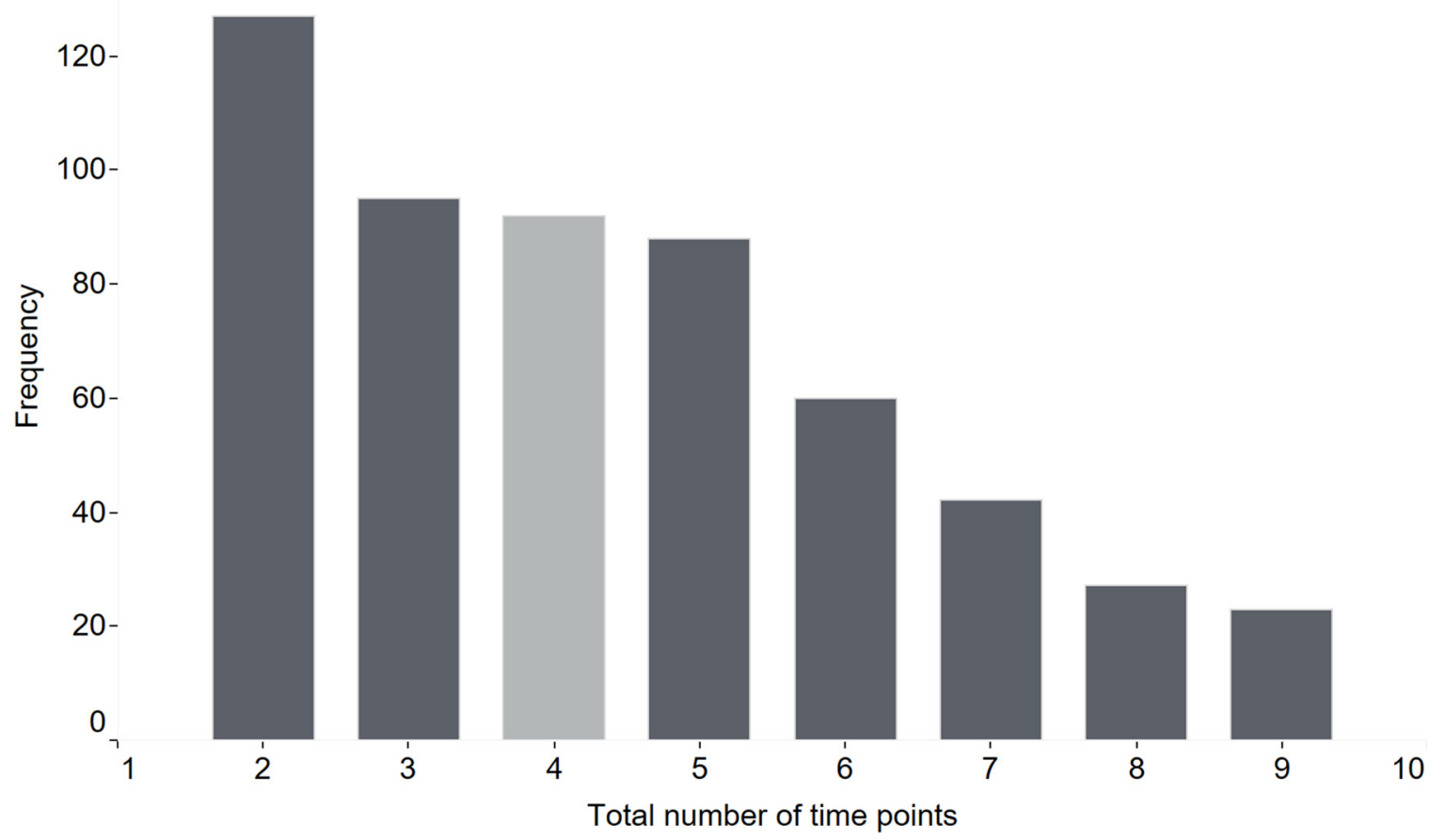

Figure 1 The frequency of the total number of time points recorded for each participant (median=4; light grey bar).

clinical and functional information at predetermined time intervals using a clinical pro forma (see details next). The first available clinical assessment at the service was taken as the baseline time point for each participant and the date of this assessment was used to determine each of the follow-up time points: 3 months, 6 months, 12 months, 2 years, 3 years, 4 years and 5 years. If no clinical notes were available within \pm 1 month of the 3 -month and 6-month time points, or \pm 3 months of the yearly time points, then this particular entry was left missing. A 'time last seen' entry was also used to capture final clinical information that did not align with one of the specified time points to ensure that every participant had data entered for the total time they were engaged with the clinical service. When data were available for a specified time point, all clinical notes from the preceding pro forma entry, up to and including the current pro forma entry were used to complete the pro forma.

\section{Clinical pro forma}

The clinical pro forma captures key clinical information about the current episode and specific illness course characteristics, and an earlier version has been used in previous studies. ${ }^{24} 29$ The pro forma collects information about: (1) basic demographics (age, gender, receipt of government benefits); (2) mental health diagnoses (based on Diagnostic and Statistical Manual of Mental Disorders (DSM-V) criteria); (3) clinical course information (hospitalisations, childhood diagnoses); (4) comorbidities (physical health diagnoses, such as autoimmune, endocrine, metabolic and so on, and suicidal thoughts and behaviours); and (5) functioning (assessed using the Social Occupational Functional Assessment
Scale (SOFAS) ${ }^{36}$ and engagement in part-time or fulltime education, employment or training, used to determine NEET status). The SOFAS is a clinician-rated measure that assesses functioning on a 0-100 scale, with lower scores suggesting more severe impairment. The instructions emphasise that the rater should aim to avoid confounding the rating with clinical symptoms.

\section{Statistical analyses}

Statistical analyses were performed using SAS Software. Overall changes in functioning (ie, 'improvement', 'no change' and 'deterioration') between baseline and time last seen were determined using a Reliable Change Index score of 10 points, and a clinically significant cut-off of equal to or above 69 was used. ${ }^{32} 34$ To characterise the pattern of change in functional impairment over time, we used Group-Based Trajectory Modelling (GBTM) using a procedure called PROC TRAJ. ${ }^{38}$ This method estimates multiple trajectory groups within the population and uses a maximum-likelihood method to calculate the probability of membership within each trajectory for each participant. We first fit the null model (one group model), and progressively increased the number of groups until we reached the optimal number of trajectory groups, which was determined using the Bayesian Information Criterion (BIC). A higher number (ie, smaller negative number) indicates a better balance between model complexity and model fit. The shape of each trajectory was examined by modelling three parameters (linear, quadratic, cubic) and then, starting with the higher order polynomials, dropping non-significant parameters from the model. If all three parameters were not significant, the linear parameter was retained. Finally, to explore which baseline factors 


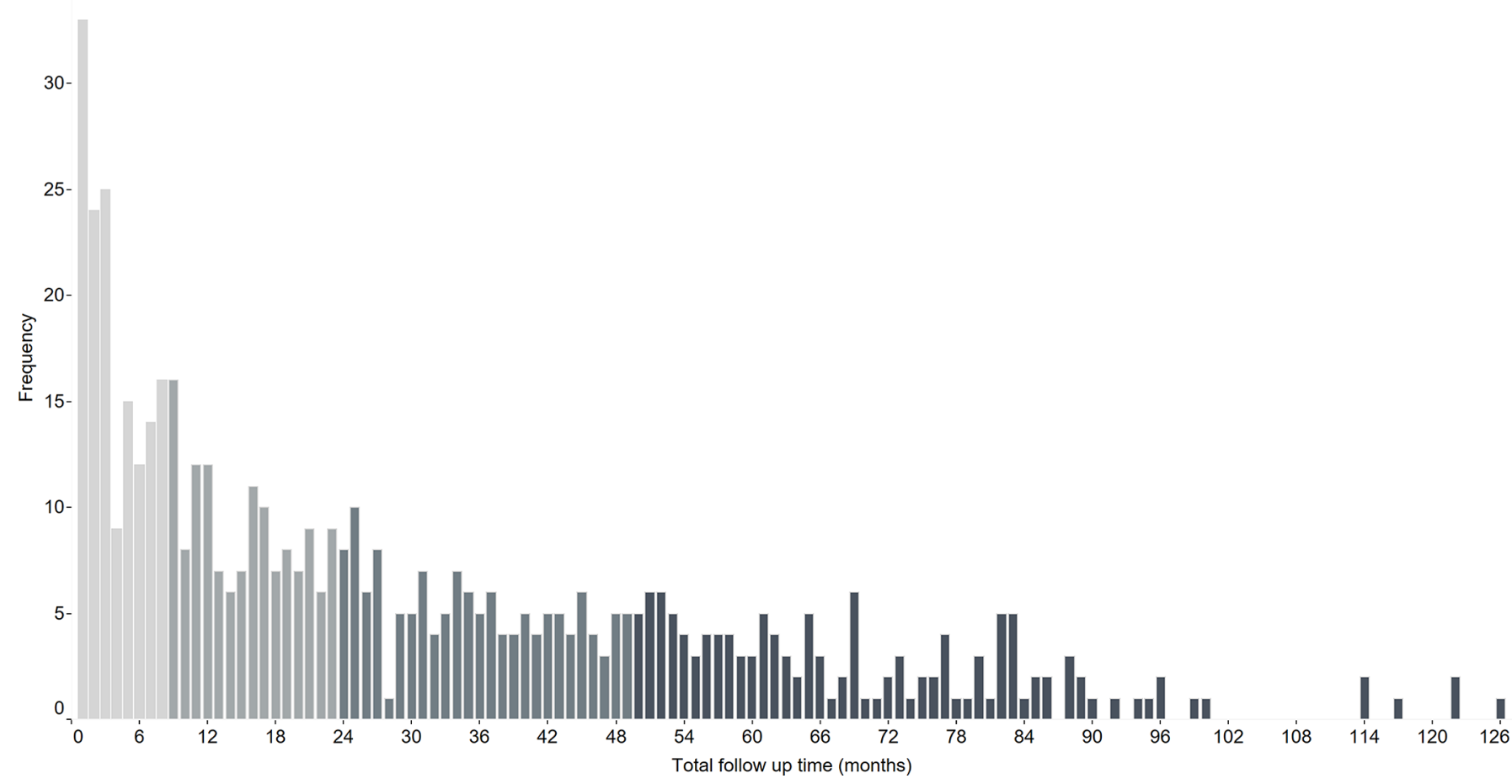

Figure 2 The distribution of the total follow-up time for each participant in months. The bars have been shaded into quartiles (median=23 months). The majority of participants (50\%) were followed up between 9 months and 49 months (ie, 4 years) after initial presentation, while $25 \%$ were followed up between 1 and 8 months, and the remaining $25 \%$ followed up between 50 months (ie, 4 years) and 126 months (ie, 10 years).

were associated with each trajectory group, we used stepwise logistic regression, which included baseline demographic and clinical characteristics: age, gender, receipt of government benefits, NEET status, mental health diagnosis, medical diagnosis, childhood mental health diagnosis, hospitalised (ever), suicide ideation (ever), suicide planning (ever) and suicide attempts (ever). An $\alpha$ level

\begin{tabular}{|c|c|c|c|}
\hline $\begin{array}{l}\text { Number of } \\
\text { groups }\end{array}$ & BIC & Null model & BIC change \\
\hline 1 & -8773.03 & 0 & - \\
\hline 2 & -8372.01 & 1 & 401.022 \\
\hline 3 & -8243.31 & 2 & 128.695 \\
\hline 4 & -8215.51 & 3 & 27.802 \\
\hline 5 & -8207.80 & 4 & 7.710 \\
\hline 6 & -8166.46 & 5 & 41.339 \\
\hline 7 & -8164.58 & 6 & 1.882 \\
\hline 8 & -8162.05 & 7 & 2.528 \\
\hline 9 & -8155.80 & 8 & 6.251 \\
\hline
\end{tabular}

Bayesian Information Criterion (BIC) change presents the changes in the BIC value as the number of trajectory group's increases. Large changes in BIC from 1 to 6 groups justified moving towards the more complex model; however, changes in BIC from 7 to 9 groups were rather small and compromised the balance between complexity and fit. Six trajectory groups were deemed to be the most parsimonious model. for entry and exclusion was set at $\mathrm{p}=0.15$ and based on the likelihood ratio statistic.

\section{RESULTS}

\section{Sample characteristics}

The sample consisted of 554 young people, 54\% $(297 / 554)$ were female and the mean age was 19.83 $(\mathrm{SD}=3.77)$. At baseline, $20 \%(113 / 554)$ identified as NEET, $17 \%$ (95/554) were currently receiving government benefits and the majority $(78 \% ; 423 / 542)$ were in the clinical range of functional impairment (ie, SOFAS score $<69)$. The most common primary diagnosis was depression $(43 \% ; 237 / 548)$, followed by bipolar disorder $(20 \% ; 108 / 548)$, and then anxiety $(18 \% ; 99 / 548)$ with comorbid mental health problems identified in $79 \%$ (428/544) of participants. Physical health comorbidities were reported in 26\% (142/554) of participants, $23 \%$ $(127 / 554)$ had previously been hospitalised due to a mental health problem and 14\% (75/554) had a mental health or behavioural diagnosis in childhood.

\section{Changes in functional impairment between baseline and time last seen}

The number of follow-up time points recorded for an individual varied between 2 and 9 (median=4) (figure 1 ) and the number of months between baseline and time last seen was between 1 and 126 (median=23 months) (figure 2). The occurrence of time last seen was spread with $38 \%$ (208/554) occurring within the first 12 months after 
Table 2 Model selection for each functional impairment trajectory group

\begin{tabular}{|c|c|c|c|c|c|}
\hline Trajectory group & Parameter & Model 1 & Model 2 & Model 3 & Model 4 \\
\hline \multirow{3}{*}{$\begin{array}{l}1 \\
\text { Serious impairment- } \\
\text { deterioration }\end{array}$} & Intercept & 51.61208 & 51.77906 & 51.21822 & 50.92215 \\
\hline & Linear & $-0.84458^{\star * *}$ & $-0.86418^{\star \star \star}$ & $-0.50281^{* * *}$ & $-0.49666^{\star \star \star}$ \\
\hline & Cubic & $-0.00022^{*}$ & -0.00022 & - & - \\
\hline \multirow{3}{*}{$\begin{array}{l}2 \\
\text { Serious impairment- } \\
\text { chronic }\end{array}$} & Intercept & 54.98897 & 54.95892 & 54.54367 & 54.75505 \\
\hline & Quadratic & 0.00966 & 0.00901 & -0.00110 & - \\
\hline & Cubic & $-0.00012^{*}$ & -0.00012 & - & - \\
\hline \multirow{3}{*}{$\begin{array}{l}3 \\
\text { Serious impairment- } \\
\text { improvement }\end{array}$} & Intercept & 41.08481 & 42.22558 & 42.03591 & 42.21444 \\
\hline & Linear & $1.76596^{\star \star \star}$ & $1.26818^{\star \star \star}$ & $1.26797^{\star \star * *}$ & $1.25871^{\star * *}$ \\
\hline & Quadratic & $-0.03534^{\star}$ & $-0.01123^{* * *}$ & $-0.01116^{\star * *}$ & $-0.01106^{* * *}$ \\
\hline \multirow{3}{*}{$\begin{array}{l}4 \\
\text { Moderate } \\
\text { impairment_chronic }\end{array}$} & Linear & 0.09497 & 0.04047 & 0.01924 & 0.02027 \\
\hline & Quadratic & -0.00309 & -0.00039 & - & - \\
\hline & Cubic & 0.00003 & - & - & - \\
\hline \multirow{4}{*}{$\begin{array}{l}5 \\
\text { Mild impairment- } \\
\text { improvement }\end{array}$} & Intercept & 67.79146 & 68.08779 & 68.12046 & 68.11021 \\
\hline & Linear & $0.46038^{\star \star \star}$ & $0.31975^{\star \star \star}$ & $0.32482^{* * *}$ & $0.32399^{\star * *}$ \\
\hline & Quadratic & $-0.01202^{*}$ & $-0.00468^{* * *}$ & $-0.00478^{\star * *}$ & $-0.00477^{\star \star *}$ \\
\hline & Cubic & 0.00009 & - & - & - \\
\hline \multirow{2}{*}{$\begin{array}{l}6 \\
\text { Slight impairment- } \\
\text { stable }\end{array}$} & Intercept & 77.35888 & 77.40056 & 77.94966 & 77.93924 \\
\hline & Linear & 0.19581 & 0.13170 & 0.04127 & 0.04153 \\
\hline
\end{tabular}

Parameter estimates are shown. Significant values are in bold. The first model identified that the cubic parameters for trajectories 3, 4, 5 and 6 were not significant and were thus dropped for model 2. Model 2 identified that the quadratic parameters for trajectories 4 and 6 were not significant, and that the cubic parameters for trajectories 1 and 2 were not significant and were dropped for model 3 . Model 3 identified that the quadratic parameter for trajectory 2 was not significant and was dropped for model 4 . The final model (model 4) had the highest Bayesian Information Criterion (BIC) and contained quadratic parameters for trajectories 1, 3 and 5 and linear parameters for trajectories 2, 4, and 6. ${ }^{*} \mathrm{P}<0.05$.

${ }^{* *} \mathrm{P}<0.01$.

${ }^{* * *} \mathrm{P}<0.001$.

baseline and $62 \%(346 / 554)$ occurring more than 1 year after baseline. Overall, between baseline and time last seen, $15 \%(79 / 538)$ had reliably deteriorated, $23 \%$ $(122 / 538)$ reliably improved and 62\% (337/538) did not reliably change, while $69 \%(370 / 538)$ were below the clinical cut-off (SOFAS $<69$ ) at time last seen.

\section{Identifying functional impairment trajectories}

GBTM identified that six distinct trajectories provided the best balance between model complexity and model fit for the data (table 1). The BIC continued to increase as the number of groups increased; however, the BIC change from seven to nine trajectories was small and resulted in trajectory groups with very small sample sizes that did not add useful information beyond that provided by the six trajectories. Table 2 shows the model selection process for the shape of each of the six trajectories. We started with all three parameters in the model (linear, quadratic and cubic). The final model (model 4) had the highest BIC and contained quadratic parameters for trajectories 1,3 and 5 and linear parameters for trajectories 2, 4 and 6 .

Figure 3 shows SOFAS score over a 5-year period for the six trajectories included in the final model (see online supplementary figure 1 for individual-level trajectories for each group). Three trajectories start out with serious functional impairment at baseline but differ in the type of change in functioning over time. The first was the second largest group of the entire sample $(29 \% ; 158 / 554)$ and included individuals who followed a chronic course of serious functional impairment with little to no change in functioning over time ('serious impairment-chronic'). The second trajectory was quadratic and included individuals who 
(6)
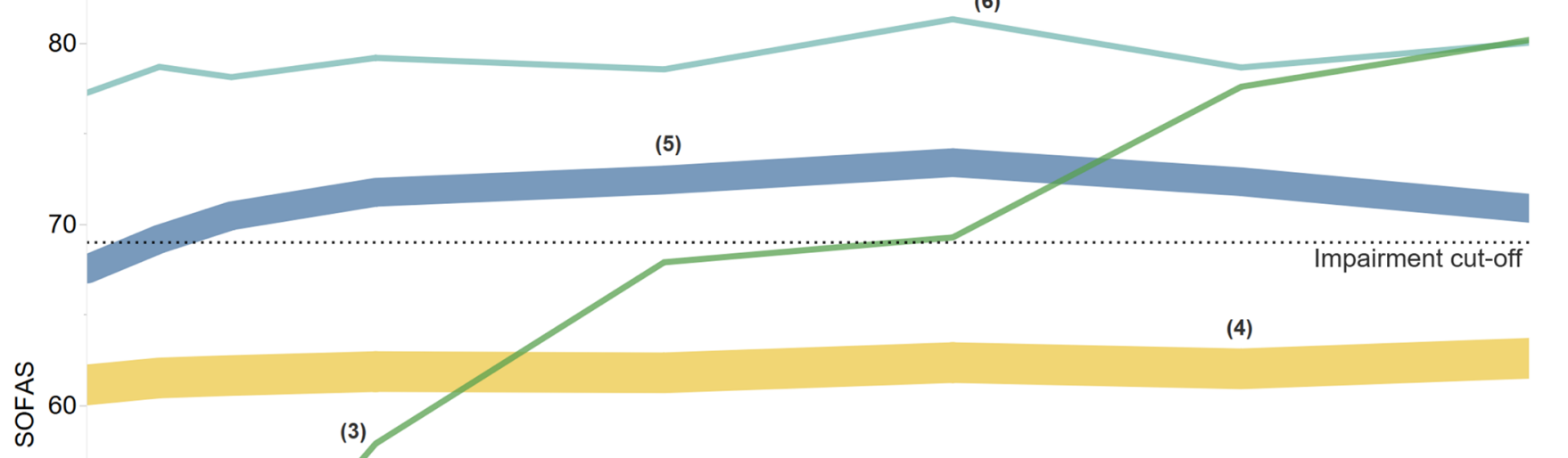

(2)

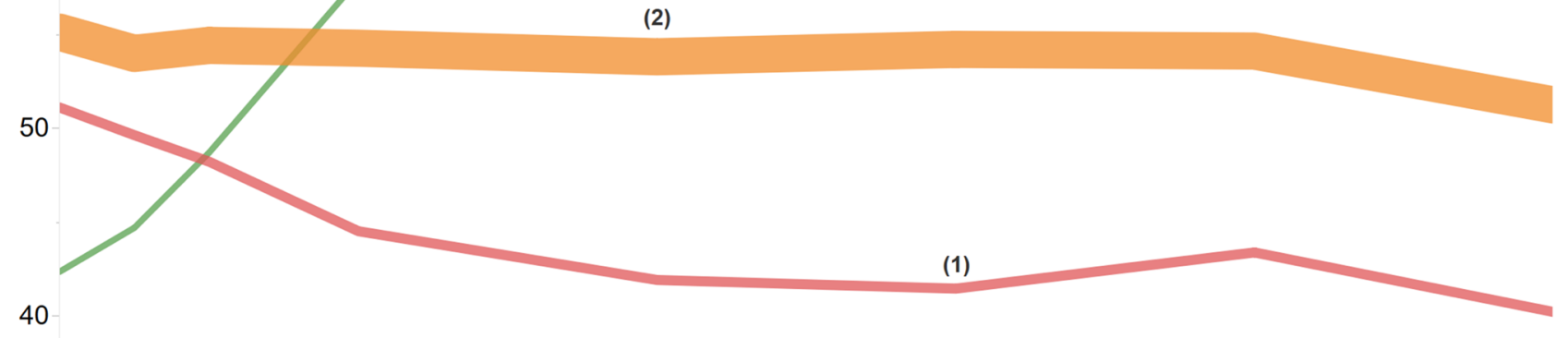

0

12

24

36

48

60

Trajectory group

(1) Serious impairment - Deterioration

(2) Serious impairment - Chronic

(3) Serious impairment - Improvement

(4) Moderate impairment - Chronic

(5) Mild impairment - Improvement

(6) Slight impairment - Stable

Figure 3 The six distinct trajectories identified for Social Occupational Functional Assessment Scale (SOFAS) score over a 5-year period. The thickness of each line represents the sample size of that particular trajectory, relative to all others. The dotted line represents the clinical impairment cut-off, which is set at a SOFAS score of 69. Slight impairment-stable ( $\mathrm{n}=24$, $4 \%$ ), intercept equal to 78 and linear trend over time; mild impairment-improvement ( $n=129,23 \%)$, intercept equal to 68 and quadratic trend over time; moderate impairment - chronic $(n=185,33 \%)$, intercept equal to 61 and linear trend over time; serious impairment-chronic ( $n=158,29 \%)$, intercept equal to 55 and linear trend over time; serious impairment-improvement $(n=19,4 \%)$, intercept equal to 42 and quadratic trend over time; serious impairment-deterioration $(n=39,7 \%)$, intercept equal to 51 and quadratic trend over time.

significantly deteriorated in the first 12 months before plateauing between 12 and 60 months ('serious impairment-deterioration'), while the third trajectory was also quadratic and included the small minority who improved significantly over the first 24 months to mild levels of functional impairment before slightly tapering off with mild to no functional impairment ('serious impairment-improvement'). By contrast, the remaining three trajectories each started out with moderate-to-mild levels of functional impairment. The first included the largest number of people across the entire sample $(33 \% ; 185 / 554)$ who presented with moderate impairment and followed a chronic course of moderate impairment over time ('moderate impairment-chronic'). The second trajectory was quadratic and characterised by individuals who were mildly impaired at baseline, but improved/functionally recovered in the first 6-12 months before tapering off and remaining in the functional recovered population over time ('mild impairment-improvement'). The final trajectory group characterised the small number of individuals who were functioning well with no more than slight impairment at baseline and whose functioning was stable over time ('slight impairment-stable').

\section{Differentiating between functional impairment trajectories}

The aim of these analyses was to identify any demographic and clinical differences at baseline between the trajectory groups. The serious impairment-chronic trajectory was chosen as the reference group because, of the most impaired groups at entry; this group was the largest group and followed a stable/chronic trajectory over time. Of the demographic and clinical variables at baseline (table 3), NEET status, age and previous hospitalisations emerged as the factors that differentiated trajectory groups and were included in the reduced model. NEET 


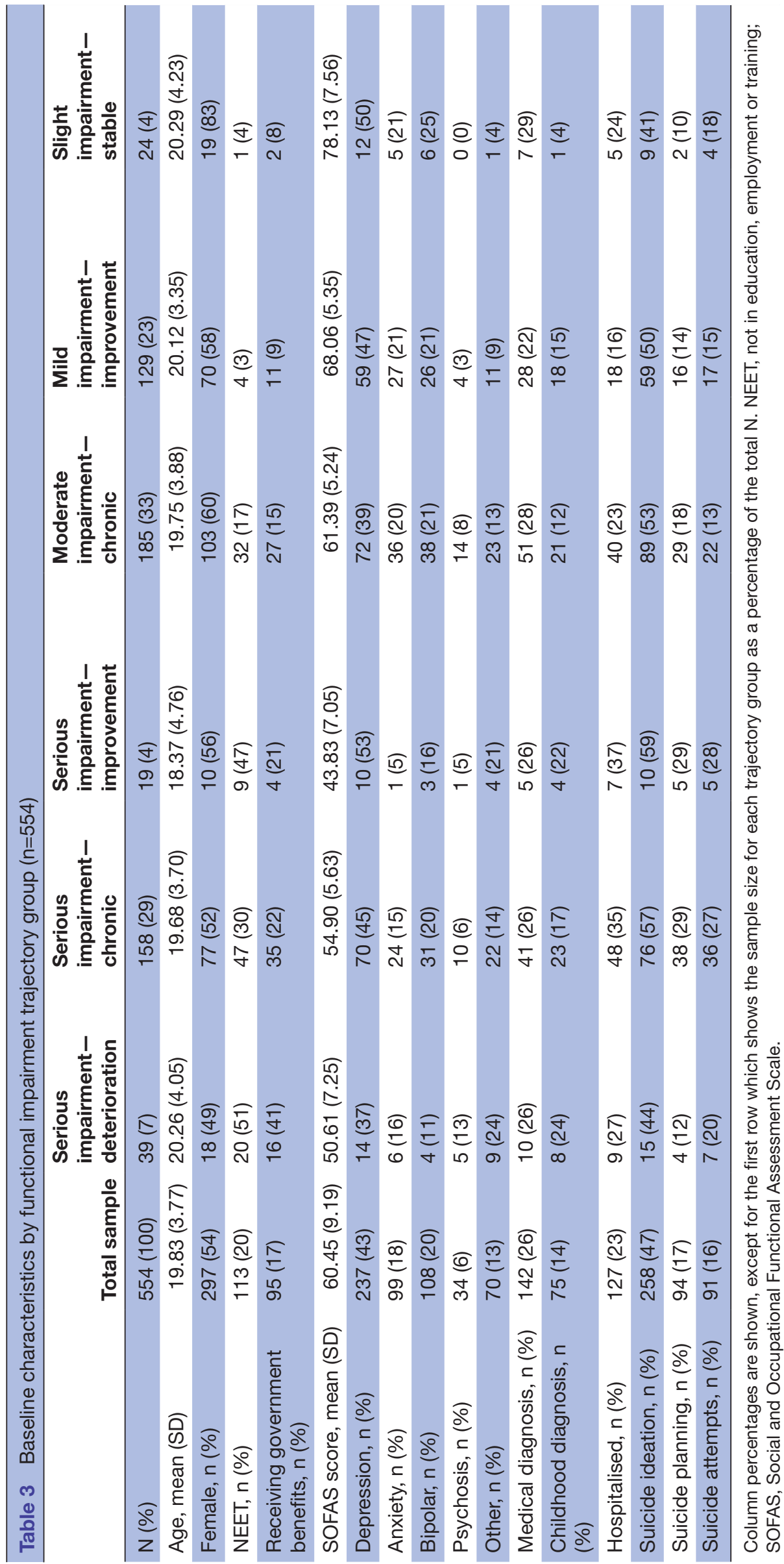


status distinguished between most trajectories, whereby those on the serious impairment-chronic trajectory were less likely to be engaged in education, employment or training compared with moderate impairmentchronic (OR $0.47,95 \%$ CI 0.27 to $0.83, \mathrm{p}<0.01$ ), mild impairment-improvement (OR $0.08,95 \%$ CI 0.03 to $0.23, \mathrm{p}<0.001)$ and slight impairment-stable (OR 0.09, $95 \%$ CI 0.01 to $0.70, \mathrm{p}<0.05)$. Regarding age, those on the serious impairment-chronic trajectory were: older than those on the serious impairment-improvement trajectory (OR $0.83,95 \% \mathrm{CI} 0.71$ to $0.98, \mathrm{p}<0.05$ ), and younger than those on the mild impairment-improvement trajectory (OR 1.08, 95\% CI 1.00 to $1.16, \mathrm{p}<0.05$ ). For previous hospitalisation, those on the serious impairment-chronic trajectory were more likely to have been previously hospitalised than those on the mild impairment-improvement trajectory (OR 2.72, 95\% CI 1.39 to $5.33, \mathrm{p}<0.01)$.

\section{DISCUSSION}

Young people with emerging mental disorders have significant functional impairment that is dynamic and chronic over the course of clinical care. Improvement occurs throughout the course of care; however, the rate of clinical impairment and functional deterioration remains high for a large number of people. The results also indicate that while individual trajectories may be highly variable, there are distinct patterns of social and occupational functioning that are differentiated by the level of functioning at entry and rate of change over the course of clinical care. Over $60 \%$ of the sample had moderate-to-serious functional impairment at entry and remained chronically impaired over time, a further $7 \%$ entered with serious impairment and deteriorated further, while approximately a quarter of the sample were mildly impaired at entry and were able to improve and functionally recover. Only a small minority $(4 \%)$, the youngest of the trajectory groups, presented with serious impairments and were able to functionally improve over time. This may reflect the benefits of early intervention; however, this requires further investigation. These distinct trajectories highlight the need for improving mental health service and individual intervention strategies to monitor and directly target these problems over the course of care to facilitate clinical, social and occupational recovery. ${ }^{10}$

The overall rate of reliable change in this study was comparable to studies conducted in similar cohorts that were followed for relatively short-term occasions of service. The rate of reliable improvement in this study (23\%) is consistent with a similar cohort of young people followed for approximately 6 months $(25 \%)^{34}$ and slightly lower than an Australian national study of young people attending headspace followed for approximately 3 months $(31 \%){ }^{32}$ Interestingly, the rate of reliable deterioration in this study was consistent with the national study at approximately $15 \%$, which suggests that deterioration occurs early and often persists over longer periods.

While the overall rate of change is important, this study examined the longer term patterns of change (ie, over a 5-year period), which were informed by multiple time points. This revealed that across all levels of impairment there were high rates of chronicity with many individuals remaining at similar levels of functioning over the course of care. For some who may have been on a path of deterioration prior to presentation for care, maintaining a consistent level of impairment may reflect a positive outcome whereby engagement with care stabilised their situation or prevented further deterioration or worsening. For others, however, not being able to return to work or education, or improve social functioning could be detrimental to their future health and socioeconomic well-being and may reflect a lack of sufficient integrated psychological and vocational interventions to directly address these outcomes. ${ }^{39} 40$

These results suggest that for those who present with mild functional impairment, functional improvement is likely to occur relatively quickly (ie, evident from the quadratic trend towards improvement within the first 6 months); however, for those with more serious impairment, there may be the need for more intensive strategies delivered over a longer period of time to prevent or address ongoing functional impairment. Previous research has shown that only a small number of young people attending these primary mental health services received specific vocational support in the previous year, ${ }^{30}$ despite evidence to suggest that adjunctive interventions targeting vocational activity can have a positive impact on functional outcomes. ${ }^{41}{ }^{42}$ Even among those with severe, comorbid disorders, early intervention combined with focused social recovery has demonstrated clinical utility over early intervention alone for improving functional outcomes. ${ }^{43}$ Together, this reiterates the need for early intervention and ongoing care that does more to directly address functional impairment over longer periods, particularly for those who present with substantial functional impairment.

For health services and clinicians, determining when to adopt these intervention strategies and for whom is critical. The general trajectories observed in this study are characterised by substantial individual variation from one time point to the next (see online supplementary figure 1). This individual variability highlights the challenge health professionals often face when planning effective long-term interventions in a cohort with emerging mental health disorders. Being NEET, previous hospitalisation and a younger age at entry was associated with the serious impairment trajectories compared with the moderate, mild and slight impairment trajectories; however, the long-term predictive utility of these characteristics is still limited. Thus, there is a need to improve health service approaches to help clinicians identify and track individual functional outcomes and trajectories over the course of care, so that the appropriate interventions can be strategically implemented. 
One solution may be the development and integration of new and emerging technologies that use routine outcome measurement and feedback within health services, to deliver more personalised interventions that respond to an individual's needs. ${ }^{445}$ Regular feedback to clinicians and individuals can provide important insights about functional impairment over time as well as the effectiveness of particular interventions for addressing key clinical and functional outcomes. ${ }^{46}$ These approaches could also make use of assessments that aim to identify underlying characteristics, such as cognition, which have demonstrated some utility in predicting changes in functioning over time. ${ }^{47-49}$

This study has some limitations. The sample used for this study focuses on individuals who were continually engaged in clinical care, which means that the overall rate of improvement or deterioration among those who disengaged is unknown. Furthermore, the overall rate of improvement and deterioration in functioning at time last seen is imperfect given that many young people may be still engaged in care and so time last seen may not align with a complete period of care. This is where the GBTM is beneficial over the overall rate of change, since it accounts for the overall trends to provide a clearer picture of change over time. While we know that this sample represents approximately $18 \%$ of the research register $(554 / 3087)$, it is unclear what proportion of the whole population attending these services this sample represents. Moreover, given that the study was conducted within the context of normal clinical service, the clinical and functional information available for particular individuals was diverse and while the option for 'not enough information available' was provided to raters, it is unclear how the type of information available impacted on the completion of the clinical pro forma. Finally, there may be other factors that account for these trajectories or differences in functional outcome that were not collected, such as, but not limited to, socioeconomic status, the type and intensity of interventions an individual received or pre-existing undiagnosed learning or developmental disorders. It is important for future work to determine the effectiveness of specific interventions on functional impairment trajectories and improving these outcomes to determine the reliability and validity of the medical file audit process used in this study.

This study provides valuable insights into the longterm functional trajectories of young people engaged in primary mental healthcare. The significant chronicity observed in this clinical cohort reiterates that ongoing functional impairment is prevalent among young people with emerging mental health disorders and should be a primary focus of intervention, in addition to symptomatic improvement. The substantial variability in individuals' trajectories over time highlights the need for better health service and individual intervention strategies that monitor and target these outcomes so that early social and occupational impairment does not result in lifetime socioeconomic burden.

Acknowledgements We would like to thank all the young people who have participated in this study, and all the staff in the Youth Mental Health Team at the Brain and Mind Centre, past and present, who have contributed to this work.

Contributors $\mathrm{FI}, \mathrm{DFH}, \mathrm{SC}$ and IBH designed the study, interpreted the results and drafted the manuscript. FI and C-AB conducted the statistical analyses. FI, NZ, AN, JG and EMS were involved in study coordination and data collection. All authors contributed to and have approved the final manuscript.

Funding This study was supported by the National Health \& Medical Research Council (NHMRC) Centre of Research Excellence grant (No. 1061043). IBH was supported by the NHMRC Research fellowship (No. 1046899). FI was supported by an Australian Postgraduate Award (APA).

Competing interests IH has been a Commissioner in Australia's National Mental Health Commission since 2012. He is the Co-Director, Health and Policy at the Brain and Mind Centre (BMC) University of Sydney. The BMC operates an early intervention youth services at Camperdown under contract to headspace. IH has previously led community-based and pharmaceutical industry-supported (Wyeth, Eli Lily, Servier, Pfizer, AstraZeneca) projects focused on the identification and better management of anxiety and depression. He is a Board Member of Psychosis Australia Trust and a member of Veterans Mental Health Clinical Reference group. He was a member of the Medical Advisory Panel for Medibank Private until October 2017. He is the Chief Scientific Advisor to, and an equity shareholder in, Innowell. InnoWell has been formed by the University of Sydney and PwC to administer the \$A30 million Australian Government Funded Project Synergy. Project Synergy is a 3-year programme for the transformation of mental health services through the use of innovative technologies. EMS is the Medical Director, Young Adult Mental Health Unit, St Vincent's Hospital Darlinghurst, Discipline Leader of Adult Mental Health, School of Medicine, University of Notre Dame, Research Affiliate, The University of Sydney and Consultant Psychiatrist. She has received honoraria for educational seminars related to the clinical management of depressive disorders supported by Servier and Eli-Lilly pharmaceuticals. She has participated in a national advisory board for the antidepressant compound Pristiq, manufactured by Pfizer. She was the National Coordinator of an antidepressant trial sponsored by Servier.

Patient consent Not required.

Ethics approval University of Sydney Human Research Ethics Committee.

Provenance and peer review Not commissioned; externally peer reviewed.

Data sharing statement No additional data available.

Open Access This is an Open Access article distributed in accordance with the Creative Commons Attribution Non Commercial (CC BY-NC 4.0) license, which permits others to distribute, remix, adapt, build upon this work non-commercially, and license their derivative works on different terms, provided the original work is properly cited and the use is non-commercial. See: http://creativecommons.org/ licenses/by-nc/4.0/

(C) Article author(s) (or their employer(s) unless otherwise stated in the text of the article) 2018. All rights reserved. No commercial use is permitted unless otherwise expressly granted.

\section{REFERENCES}

1. Walker ER, McGee RE, Druss BG. Mortality in mental disorders and global disease burden implications: a systematic review and metaanalysis. JAMA Psychiatry 2015;72:334-41.

2. Whiteford HA, Degenhardt L, Rehm J, et al. Global burden of disease attributable to mental and substance use disorders: findings from the Global Burden of Disease Study 2010. The Lancet 2013;382:1575-86.

3. Vos T, Barber RM, Bell B, et al. Global, regional, and national incidence, prevalence, and years lived with disability for 301 acute and chronic diseases and injuries in 188 countries, 1990-2013: a systematic analysis for the Global Burden of Disease Study 2013. Lancet 2015;386:743-800.

4. Goodman A, Joyce R, Smith JP. The long shadow cast by childhood physical and mental problems on adult life. Proceedings of the National Academy of Sciences 2011;108:6032-7.

5. Merikangas KR, He JP, Burstein M, et al. Lifetime prevalence of mental disorders in U.S. adolescents: results from the National 
Comorbidity Survey Replication--Adolescent Supplement (NCS-A). J Am Acad Child Adolesc Psychiatry 2010;49:980-9.

6. Gibb SJ, Fergusson DM, Horwood LJ. Burden of psychiatric disorder in young adulthood and life outcomes at age 30 . Br J Psychiatry 2010;197:122-7.

7. Copeland WE, Wolke D, Shanahan L, et al. Adult functional outcomes of common childhood psychiatric problems: a prospective, longitudinal study. JAMA Psychiatry 2015;72:892-9.

8. Trautmann S, Rehm J, Wittchen Hans-Ulrich. The economic costs of mental disorders. EMBO Rep 2016;17:1245-9.

9. Bloom D, Cafiero E, Jané-Llopis E, et al. The global economic burden of noncommunicable diseases: Program on the Global Demography of Aging, 2012.

10. Scott J, Fowler D, McGorry P, et al. Adolescents and young adults who are not in employment, education, or training: British Medical Journal Publishing Group, 2013.

11. Coryell W, Scheftner W, Keller M, et al. The enduring psychosocial consequences of mania and depression. Am J Psychiatry 1993;150:720-7.

12. Furukawa TA, Takeuchi $\mathrm{H}$, Hiroe $\mathrm{T}$, et al. Symptomatic recovery and social functioning in major depression. Acta Psychiatr Scand 2001;103:257-61.

13. Kennedy N, Abbott R, Paykel ES. Remission and recurrence of depression in the maintenance era: long-term outcome in a Cambridge cohort. Psychol Med 2003;33:827-38.

14. Riihimäki KA, Vuorilehto MS, Melartin TK, et al. Five-year outcome of major depressive disorder in primary health care. Psychol Med 2014;44:1369-79.

15. Judd LL, Schettler PJ, Solomon DA, et al. Psychosocial disability and work role function compared across the long-term course of bipolar I, bipolar II and unipolar major depressive disorders. J Affect Disord 2008;108:49-58.

16. Riihimäki K, Vuorilehto $\mathrm{M}$, Isometsä E. A 5-year prospective study of predictors for functional and work disability among primary care patients with depressive disorders. Eur Psychiatry 2015;30:51-7.

17. Vitiello B, Rohde P, Silva S, et al. Functioning and quality of life in the Treatment for Adolescents with Depression Study (TADS). J Am Acad Child Adolesc Psychiatry 2006;45:1419-26.

18. Wagner KD, Ambrosini P, Rynn M, et al. Efficacy of sertraline in the treatment of children and adolescents with major depressive disorder: two randomized controlled trials. JAMA 2003;290:1033-41.

19. Brent DA, Holder D, Kolko D, et al. A clinical psychotherapy trial for adolescent depression comparing cognitive, family, and supportive therapy. Arch Gen Psychiatry 1997;54:877-85.

20. Peters AT, Jacobs RH, Feldhaus C, et al. Trajectories of functioning into emerging adulthood following treatment for adolescent depression. J Adolesc Health 2016;58:253-9.

21. Hodgekins J, Birchwood M, Christopher R, et al. Investigating trajectories of social recovery in individuals with first-episode psychosis: a latent class growth analysis. Br J Psychiatry 2015;207:536-43.

22. Santesteban-Echarri O, Paino M, Rice S, et al. Predictors of functional recovery in first-episode psychosis: A systematic review and meta-analysis of longitudinal studies. Clin Psychol Rev 2017:58:59-75.

23. Fowler D, Hodgekins J, Painter M, et al. Cognitive behaviour therapy for improving social recovery in psychosis: a report from the ISREP MRC Trial Platform Study (Improving Social Recovery in Early Psychosis). Psychol Med 2009;39:1627-36.

24. Hickie IB, Scott EM, Hermens DF, et al. Applying clinical staging to young people who present for mental health care. Early Interv Psychiatry 2013;7:31-43.

25. McGorry PD, Hickie IB, Yung AR, et al. Clinical staging of psychiatric disorders: a heuristic framework for choosing earlier, safer and more effective interventions. Aust N Z J Psychiatry 2006;40:616-22.

26. McGorry P, Bates T, Birchwood M. Designing youth mental health services for the 21st century: examples from Australia, Ireland and the UK. Br J Psychiatry Suppl 2013;54:s30-s35.

27. Scott J, Scott EM, Hermens DF, et al. Functional impairment in adolescents and young adults with emerging mood disorders. $\mathrm{Br} \mathrm{J}$ Psychiatry 2014;205:362-8.
28. O'Dea B, Glozier N, Purcell R, et al. A cross-sectional exploration of the clinical characteristics of disengaged (NEET) young people in primary mental healthcare. BMJ Open 2014;4:e006378.

29. Scott EM, Hermens DF, Glozier N, et al. Targeted primary carebased mental health services for young Australians. Med J Aust 2012;196:136-40.

30. O'Dea B, Lee RS, McGorry PD, et al. A prospective cohort study of depression course, functional disability, and NEET status in help-seeking young adults. Soc Psychiatry Psychiatr Epidemiol 2016;51:1395-404

31. Judd LL, Akiskal HS, Zeller PJ, et al. Psychosocial disability during the long-term course of unipolar major depressive disorder. Arch Gen Psychiatry 2000;57:375-80.

32. Rickwood DJ, Mazzer KR, Telford NR, et al. Changes in psychological distress and psychosocial functioning in young people visiting headspace centres for mental health problems. Med J Aust 2015;202:537-42.

33. Cross SP, Hermens DF, Hickie IB. Treatment patterns and short-term outcomes in an early intervention youth mental health service. Early Interv Psychiatry 2016;10:88-97.

34. Cross SP, Scott J, Hermens DF, et al. Clinical outcomes for youth with subthreshold severe mental disorders accessing an early intervention service. Psychiatric Services In Press.

35. McGorry PD, Tanti C, Stokes R, et al. headspace: Australia's National Youth Mental Health Foundation--where young minds come first. Med J Aust 2007;187:S68.

36. Goldman HH, Skodol AE, Lave TR. Revising axis V for DSMIV: a review of measures of social functioning. Am J Psychiatry 1992:149:1148-56.

37. Falkenström F. Does psychotherapy for young adults in routine practice show similar results as therapy in randomized clinical trials? Psychother Res 2010;20:181-92.

38. Nagin DS, Odgers CL. Group-based trajectory modeling in clinical research. Annu Rev Clin Psychol 2010;6:109-38.

39. Power E, Clarke M, Kelleher I, et al. The association between economic inactivity and mental health among young people: a longitudinal study of young adults who are not in employment, education or training. Ir J Psychol Med 2015;32:155-60.

40. Rodwell L, Romaniuk H, Nilsen W, et al. Adolescent mental health and behavioural predictors of being NEET: a prospective study of young adults not in employment, education, or training. Psychol Med 2017:1-11.

41. Burns T, Catty J, Becker T, et al. EQOLISE Group. The effectiveness of supported employment for people with severe mental illness: a randomised controlled trial. Lancet 2007:370:1146-52.

42. Drake RE, McHugo GJ, Bebout RR, et al. A randomized clinical trial of supported employment for inner-city patients with severe mental disorders. Arch Gen Psychiatry 1999;56:627-33.

43. Fowler D, Hodgekins J, French P, et al. Social recovery therapy in combination with early intervention services for enhancement of social recovery in patients with first-episode psychosis (SUPEREDEN3): a single-blind, randomised controlled trial. Lancet Psychiatry 2018:5:41-50.

44. Boswell JF, Kraus DR, Miller SD, et al. Implementing routine outcome monitoring in clinical practice: benefits, challenges, and solutions. Psychother Res 2015;25:6-19.

45. Cross SP, Hickie I. Transdiagnostic stepped care in mental health Public Health Res Pract 2017;27.

46. Carlier IV, Meuldijk D, Van Vliet IM, et al. Routine outcome monitoring and feedback on physical or mental health status: evidence and theory. J Eval Clin Pract 2012;18:104-10.

47. Lee RSC, Hermens DF, Scott J, et al. A transdiagnostic study of education, employment, and training outcomes in young people with mental illness. Psychol Med 2017;47:2061-70.

48. Iorfino F, Hickie IB, Lee RS, et al. The underlying neurobiology of key functional domains in young people with mood and anxiety disorders: a systematic review. BMC Psychiatry 2016;16:1.

49. Lee RS, Hermens DF, Redoblado-Hodge MA, et al. Neuropsychological and socio-occupational functioning in young psychiatric outpatients: a longitudinal investigation. PLoS One 2013;8:e58176. 